\title{
Protocollo della seduta ordinaria della Camera medica svizzera
}

\author{
Giovedì, 19 maggio 2005, dalle 9.30 alle 17.00, Centro dei congressi bea bern congress, Berna
}

Annamaria Müller Imboden, segretaria generale FMH

Nota della redattrice del protocollo: per facilitare la lettura i punti all'ordine del giorno vengono riportati secondo l'ordine del giorno e non nell'ordine della discussione, che è però indicata tra parentesi quadre [].

Inizio della riunione: 9:40

\section{Benvenuto, comunicazioni, costituzione dell'ufficio}

Jacques de Haller, presidente della FMH, dà il benvenuto ai presenti, li ringrazia della loro presenza e costata che il quorum è raggiunto e che quindi l'assemblea è regolarmente costituita. Seguono osservazioni d'ordine organizzativo e amministrativo sullo svolgimento della giornata. A causa di diverse indisponibilità l'ordine dei punti all'ordine del giorno subisce qualche modifica. Jacques de Haller saluta in particolare il nuovo responsabile della divisione Comunicazione, Daniel Lüthi e gli offre la possibilità di presentarsi brevemente e di esporre le sue riflessioni sul ruolo e sull'importanza della comunicazione in seno alla FMH. Dopo di che il presidente rende omaggio a Reto Steiner, il portavoce della FMH, andato in pensione.

\section{Vengono designati come scrutatori}

Valentin Audétat, Armand Bottani, Giorgio Bugliani, Peter Buser, Robert Cougn, Jörg Fritschi, Jean-Pierre Grob, Niklaus Hasler, Reto Ludwig, Rolf Schlumpf, Jürg Schwegler.

\section{Rapporto annuale 2004 della FMH}

Il rapporto annuale della FMH previsto dallo statuto, integrato nel rapporto di gestione, è stato pubblicato come allegato nel Bollettino dei medici svizzeri N. 18 del 4 maggio 2004. Il rapporto non suscita nessuna osservazione speciale.

La proposta 2/1 (Comitato centrale)

che sollecita l'approvazione del rapporto annuale, viene approvata all'unanimità senza voti contrari e senza astensioni.

\section{Conti annuali 2004 della FMH}

Annamaria Müller Imboden, la segretaria centrale, presenta i risultati principali dello scorso anno d'esercizio che, ancora una volta, chiude con una notevole eccedenza delle entrate, proveniente principalmente dalle entrate straordinarie del processo vinto, da un migliore rendimento del perfezionamento professionale e dalla riduzione delle uscite relative al censimento del valore intrinseco. È previsto di destinare retroattivamente la parte delle eccedenze di entrate relativa al perfezionamento professionale alle riserve della formazione, del perfezionamento professionale e dell'aggiornamento. Il rappresentante dell'organo di controllo PWC, Heribert Riesterer, conferma che i conti sono stati tenuti in modo corretto e raccomanda che vengano approvati. Dopo che sono state chiarite alcune questioni relative alla controversia giudiziaria vinta, allo sponsoring e alle partecipazioni finanziarie, la Camera passa alle votazioni.

\subsection{Approvazione}

La proposta N. 3.1/1 (Comitato centrale) che chiede l'approvazione dei conti annuali 2004, compresi i conti del «Fondo di assistenza» e del «Fondo per il perfezionamento professionale dei medici svizzeri nei paesi in via di sviluppo» viene approvata all'unanimità senza astensioni o voti contrari. Si ringrazia per la presentazione trasparente dei conti.

Christoph Ramstein, presidente del VEDAG, ringrazia i membri del Comitato centrale e l'insieme dei collaboratori della segreteria generale per il lavoro svolto, rendendo loro omaggio a nome dei presenti.

\subsection{Scarico al Comitato centrale}

\section{La proposta N. 3.2/1 (Comitato centrale)}

invita la Camera medica svizzera a seguire le raccomandazioni dell'organo di controllo. La Camera vota all'unanimità lo scarico ai membri del Comitato centrale per la sua gestione dell'esercizio 2004, senza voti contrari o astensioni. 


\section{Budget 2006 della FMH, determinazione della quota di membro per il 2006}

Annamaria Müller Imboden presenta il budget per il 2006. Fa notare in particolare la transizione in corso dal budget tradizionale al budget strategico, che causerà per la Camera medica dei cambiamenti che concernono la pianificazione, la documentazione e la deliberazione. Il budget 2006 viene presentato come budget equilibrato, con i contributi membri che rimangono uguali all'anno precedente. Le opzioni strategiche prevedono il mantenimento della formazione, del perfezionamento professionale e dell'aggiornamento, il posizionamento della divisione Dati, Demografia e Qualità (DDQ), delle tariffe e l'ampliamento del dipartimento Comunicazione. Inoltre, è previsto di intensificare la cooperazione internazionale e di mettere un accento sui contatti nazionali. Anche per quel che concerne la gestione finanziaria e la stesura dei conti sono previsti dei cambiamenti, che non interessano però il budget in sé.

Per quel che concerne le proposte presentate alla Camera medica, la segretaria generale osserva quanto segue: una proposta della SVM (Société Vaudoise de médecine) è stata ritirata poco prima dell'inizio della seduta, ma figura ancora nella documentazione, come anche la controproposta del Comitato centrale, che non ha più quindi neanche essa - ragione di essere. Per l'HIN viene chiesto alla Camera medica di rinviare questo punto a più tardi, perché il finanziamento delle prestazioni HIN sarà esaminato nel corso dell'estate e sottoposto alla Camera medica svizzera straordinaria prevista per il mese di dicembre. Le altre due proposte relative al contributo speciale saranno discusse se i delegati alla Camera medica auspicano che in futuro siano integrati nei contributi di base. Il Comitato centrale è favorevole a questa proposta e ne terrà conto nel budget 2007.

La proposta N. 4/1 (Comitato centrale)

che chiede l'approvazione del budget presentato per il 2006 viene approvata con 1 voto contrario e senza astensioni, e con i contributi seguenti:

Contributi dei membri 2006

\begin{tabular}{llll} 
Categoria di contributo 1 & Medici con attività indipendente & Fr. 530.- \\
Categoria di contributo 2 & Medici dipendenti con funzione dirigenziale & Fr. 530.- \\
Categoria di contributo 3 & $\begin{array}{l}\text { Medici dipendenti con funzione non dirigenziale e che } \\
\text { non seguono nessun perfezionamento professionale } \\
\text { della FMH }\end{array}$ & Fr. 265.- \\
\hline Categoria di contributo 4 & Medici in perfezionamento professionale & Fr. 265.- \\
\hline Categoria di contributo 5 & Medici con residenza e attività professionale all'estero & Fr. 132.- \\
Categoria di contributo 6 & Membri temporaneamente non attivi come medici & Fr. 132.-
\end{tabular}

La proposta N. 4/2 (Marc Müller, CMB)

Richiede un nuovo prolungamento per tre anni del sostegno finanziario per l'assistenza in studio medico di membri FMH in perfezionamento professionale, sotto forma di un contributo speciale di Fr. 25.- per membro, valido per le categorie di contributo 1, 2, 3 e 4. Per il contributo speciale è necessaria ogni anno l'approvazione del budget. Questa proposta viene giustificata con il fatto che la possibilità di perfezionamento professionale nello studio medico è molto apprezzata e che gli interessati lo considerano indispensabile. L'assistenza nello studio medico costituisce una misura molto importante perché offre alle nuove generazioni di medici un approccio interessante alla professione. Aumentano le possibilità che il progetto pilota stia per essere trasformato in una forma regolare del perfezionamento professionale, anche perché l'ambiente politico vi si interessa sempre di più. In considerazione di quanto appena detto la proposta viene approvata con 1 voto contrario e 6 astensioni.

\section{La proposta N. 4/3 (Comitato centrale)}

sollecita nuovamente per il 2006 il mantenimento del contributo speciale per il finanziamento del consolidamento nazionale dei dati dell'importo di Fr. 20.- per membro, valido per le categorie 1 e 2 . Il fatto che si debbano mettere a disposizione dei mezzi per raccogliere e elaborare i dati non viene contestato, ma la Camera medica si chiede, tuttavia, perché questo finanziamento non venga integrato nei costi ordinari della FMH. Il Comitato centrale risponde che questa integrazione verrà fatta non appena saranno conclusi i lavori preliminari per i quali sono necessari mezzi speciali. La Camera comprende la logica di questi argomenti e approva il contributo con 2 voti contrari e 5 astensioni.

\section{La proposta N. 4/4 (Comitato centrale)}

è una proposta poco abituale, in quanto auspica che sul finanziamento delle prestazioni HIN per tutti i membri della FMH si decida solamente nell'ambito della seduta straordinaria della Camera medica che si terrà il 2 dicembre 2005. Dopo brevi chiarimenti da parte del Comitato centrale sull'impossibilità di presentare alla Camera nei tempi a disposizione un progetto concreto accompagnato da una proposta seria di finanziamento, i delegati approvano la proposta senza voti contrari e con 5 astensioni. Al Comitato centrale viene espressa la speranza che alla fine dell'anno non presenti semplicemente una (nuova) richiesta di prolungamento. 


\section{Formazione di base, perfezionamento professionale e aggiornamento continuo FPFA}

Max Giger, il responsabile del settore formazione medica (Medical Education), presenta brevemente la situazione attuale e gli sviluppi nel campo della formazione di base, del perfezionamento professionale e dell'aggiornamento. I punti principali sono costituiti dall'accreditamento della FMH, dalla creazione di titoli federali di medico specialista nonché dai lavori concernenti la legge federale sulle professioni mediche (LPMed). Il dipartimento competente non è sempre dello stesso parere della FMH, ma si è fiduciosi che riusciranno a trovare delle soluzioni costruttive per i diversi punti.

\subsection{Creazione di un titolo di medico specialista in chirurgia della mano}

Proposta N. 5.1/1 (Comitato centrale)

Alla Camera medica viene proposta la creazione di un titolo di specialista in chirurgia della mano. Si intende sostenere così gli sforzi per una chirurgia della mano competitiva, d'alto valore qualitativo e ben rappresentata quantitativamente. La CPA è favorevole alla creazione di questo titolo, ma non lo sono - invece - tutte le società mediche specialistiche interessate. Si criticano principalmente la mancanza di compatibilità con l'UE, i doppioni possibili nei programmi di perfezionamento professionale e i problemi relativi alla garanzia dei diritti acquisiti (valore intrinseco). Viene sollevata la questione su quali sarebbero le conseguenze se il capodipartimento competente respingesse la creazione di questo titolo di specialista. Il Comitato centrale è del parere che le questioni d'ordine tariffario non debbano essere preposte a quelle relative alla qualità. Competente per la creazione di un titolo federale è chiaramente la Confederazione e la FMH non intende impegnarsi su due vie (non creerà dei titoli FMH in parallelo ai titoli federali). Tenendo presenti tutti questi punti di vista la Camera approva la proposta con 9 voti contrari e 19 astensioni.

\subsection{Commissione per il perfezionamento professionale e l'aggiornamento (CPA)}

\section{a) Seggio della Società svizzera dei medici omeopatici (SSMO)}

La proposta N. 5.2.a/1 (Bruno Ferroni, SSMO) chiede che l'associazione svizzera dei medici omeopatici (SSMO) entri nella Commissione per il perfezionamento professionale e l'aggiorna- mento (CPA). I presupposti sono dati, poiché in virtù dell'art. 41 cpv. 4 degli statuti della FMH, le associazioni mediche responsabili di una formazione approfondita o di attestati di formazione complementare, possono far parte della CPA. La proposta viene approvata con 76 voti favorevoli, 47 contrari e 21 astensioni.

\section{b) Seggio della Società svizzera per la terapia neurale (terapia di regolazione) secondo Huneke (SMSTN)}

\section{Proposta N. 5.2.b/1 (Andreas Beck, SMSTN)}

Analogamente alla proposta 5.2.a/1 con questa proposta si chiede l'adesione della Società svizzera per la terapia neurale (terapia di regolazione) secondo Huneke (SMSTN) alla Commissione per il perfezionamento professionale e l'aggiornamento. Anche questa proposta viene approvata con 76 voti favorevoli, 47 contrari e 28 astensioni.

\section{Attività politiche}

Il consigliere nazionale Yves Guisan, vicepresidente della FMH, fa una panoramica delle principali consultazioni parlamentari di interesse per la FMH. Concretamente si tratta dei punti seguenti:

- La procedura di consultazione relativa al blocco dell'accesso alla pratica privata (Ordinanza del 3 luglio 2002 sulla limitazione del numero di fornitori di prestazioni ammessi ad esercitare la propria attività a carico dell'assicurazione obbligatoria contro le malattie): sul blocco dell'accesso alla pratica privata la FMH è stata ricevuta in udienza, ha espresso la sua disapprovazione di massima, ha presentato i punti da lei criticati, e ha fatto appello alla responsabilità della Confederazione per quel che concerne gli effetti possibili di questo blocco sulla copertura delle cure mediche.

- La Legge federale sulle professioni mediche: in occasione delle consultazioni relative alla Legge federale sulle professioni mediche si è appreso con soddisfazione che per il perfezionamento professionale è previsto che sia competente una sola organizzazione. Positiva è anche la solidarietà con altre organizzazioni professionali e le università. Meno positivo è, invece, il rifiuto del «diritto al perfezionamento professionale». Un altro problema, ancora poco noto ma che si sta inasprendo sempre di più rischia di diventare l'assicurazione responsabilità civile profes- 
sionale obbligatoria, perché per i medici diventa sempre più difficile stipulare delle polizze assicurative finanziarmente sostenibili. Molti assicuratori di responsabilità civile classificano il corpo medico come gruppo a rischio elevato con le relative conseguenze contrattuali.

- La revisione della LAMal e il suo orientamento: la Commissione del Consiglio degli Stati si occuperà di questioni relative al finanziamento degli ospedali e alla libertà contrattuale.

In generale si sta consolidando e professionalizzando il lavoro di lobbying della FMH in parlamento.

Nella discussione che segue la Camera medica esprime i suoi vivi ringraziamenti alla FMH per il suo intervento, deplorando contemporaneamente che si tenga apparentemente poco conto delle richieste del corpo medico. Le società cantonali mediche vengono invitate a documentare in modo preciso la penuria di medici e di renderla nota alle autorità cantonali competenti. Per finire rientra nelle competenze dei cantoni applicare il blocco delle ammissioni.

\section{La proposta N. 6/1 (Hans Kurt, FMPP)}

che prega il Comitato centrale di fare di tutto affinché venga soppresso il blocco delle ammissioni alla pratica privata o per lo meno affinché possa venir precisato e risolto il problema del lavoro a tempo parziale e dell'interruzione dell'attività medica, viene trasmessa sotto forma di mandato al Comitato centrale e da questi prontamente approvata.

\section{Tariffe e contratti}

Franco Muggli, responsabile del settore tariffario e della «Task-Force» Tariffe del Comitato centrale, dà qualche informazione sul tema TARMED, dopo aver cordialmente ringraziato tutte le persone implicate. Nei corso dei lavori e delle trattative ci si è resi conto di quanto sia importante disporre di dati propri, tanto più che la tariffa, che all'origine rientrava nella gestione aziendale, è passata ora al settore dell'economia pubblica e che la minaccia di un budget globale per le cure ambulatoriali non è lontano. Ancora aperte e non risolte continuano ad essere le questioni sui parametri e sui criteri di efficacia e adeguamento. Ciò nonostante la pressione politica in vista di un controllo dei costi attraverso una meccanica tariffaria è rimasta intatta. Con esempi molto chiari Franco Muggli spiega che solamente «avere i dati» non è ancora sufficiente, ma i risultati devono essere interpretati con grande cura e - una volta considerati da vicino - devono dare un'immagine sempre più differenziata. Attualmente, con il progetto «NAKO» si sta realizzando e consolidando la gestione dei dati. Questo è importante tanto più che l'elaborazione e la conclusione dei cosiddetti «contratti susseguenti TARMED» procede a pieno ritmo. Infine, Franco Muggli fa notare la complessa e lunga procedura relativa alle trattative e alle decisioni negli organi tariffari e conta sulla comprensione da parte di tutti se le proposte delle società mediche hanno ritardi in parte notevoli e subiscono anche delle «diluizioni» di contenuto. Come si sa, le negoziazioni tariffarie non sono dei «contratti di fornitura».

Res Haefeli, presidente uscente del G7, presenta la situazione per quel che concerne la regolamentazione che seguirà la neutralità dei costi. Un mese prima della fine della fase di neutralità dei costi, ovvero prima che vengano fissati i valori definitivi del punto tariffario, i lavori avanzano a pieno ritmo. Diversi punti devono essere ancora chiariti e corretti, tra l'altro anche importanti inesattezze nella banca dei dati di santésuisse. Un punto delicato concerne il trattamento del volume di compensazione, che si misura secondo i volumi «di troppo» ovvero «non sfruttati» dei cantoni o delle regioni di valore del punto tariffario, volume risultante dalla differenza rispetto al valore del punto tariffario neutro per quel che concerne i costi costatato in modo retroattivo. Alcuni cantoni devono rendere degli importi enormi, altri hanno - invece - ancora qualcosa «a loro favore». Sulla base dei volumi di correzioni verranno fissati anche i valori intermedi e quelli definitivi del punto. In una conferenza dei presidenti cantonali che sarà tenuta dopo la Camera medica si dovrà discutere in modo approfondito di tutti questi problemi si deciderà sul modo concreto di procedere. Si tratta in particolare della scottante questione relativa all'armonizzazione del valore del punto tariffario. È un tema di cui si è parlato in modo molto accesso nel corso della discussione che è seguita alle spiegazioni di Res Haefeli. Il principio di per sé non è contestato, ma i pareri sono divergenti sulla via da seguire e sul calendario da rispettare. È stata pure criticata l'assenza del settore ambulatoriale ospedaliero. Qui, però, da parte della FMH non si può intraprendere niente, in quanto delle trattative è competente l'associazione degli ospedali. Per finire Res Haefeli informa anche sull'accordo previsto concernente prestazioni e costi, che dovrebbero sostituire l'accordo sulla neutralità dei costi. Contraria- 
mente a quest'ultimo, la nuova convenzione non conterrà nessuna direttiva vincolante, ma solo delle raccomandazioni alle società mediche cantonali, alle quali - una volta terminata la fase della neutralità dei costi - spetta nuovamente di condurre le trattative. L'obiettivo è di creare delle regolamentazioni che siano il più possibile uniformi e partneriali per raggiungere l'obiettivo, cioè per determinare i valori del punto tariffario, ed evitare di dover ricorrere alle istanze di ricorso. Tuttavia, non è possibile sfuggire all'influenza dell'incaricato alla sorveglianza dei prezzi, che deve essere obbligatoriamente consultato dai governi cantonali nell'ambito della procedura di approvazione e/o di fissazione del valore del punto. Pure problematica appare la convergenza auspicata, se le convenzioni vengono poi applicate esclusivamente a livello cantonale e in modo separato per l'esercizio della professione nello studio medico e le cure ambulatoriali in ospedale. Ma questa è la situazione sul piano legale. Dopo una discussione animata, viene ringraziato cordialmente Res Haefeli per il lavoro svolto.

Franco Muggli presenta rapidamente la situazione nel settore della LAINF. Qui la fase dedicata alla stabilità dei costi si è conclusa da molto tempo e il valore del punto tariffario è fissato in modo definitivo. Sostenuta dall'UFSP, la CTM fa adesso pressione per la conclusione di una convenzione contenente un nuovo pilotaggio dei costi di carattere permanente. Nel frattempo il fatto è che esistono delle convenzioni in vigore non denunciate e che la Confederazione non dispone di nessun potere decisionale a livello giuridico.

Olivier Kappeler, membro del Comitato centrale incaricato del settore del valore intrinseco, informa sulla situazione attuale della banca dati del valore intrinseco, che si prevede ora di mettere in servizio nel corso della seconda metà dell'anno. Gli affari in sospeso vengono trattati conformemente al calendario previsto e dovrebbero essere conclusi entro i termini previsti, sempre che i medici coinvolti vi partecipino in modo costruttivo e rapido. Parallelamente, in accordo con santésuisse, la FMH ha inoltrato una domanda presso la Commissione della concorrenza. Questa domanda comprende due questioni: il diritto dei cartelli è applicabile nel settore del valore intrinseco? Un'approvazione in virtù del diritto federale sostituisce, eventualmente, le basi legali mancanti? Viene confermato che la domanda è stata ricevuta, ma la FMH attende ancora una risposta.
La proposta N. 7/1 (Jürg Schlup, Berna) domanda al Comitato centrale che cosa ha intenzione di fare quando la convenzione tariffaria per le prestazioni paramediche nello studio medico sarà scaduta e che cosa consiglierà ai membri della FMH. La situazione giuridica non è per niente chiara. Hanspeter Kuhn, vicesegretario generale della FMH, indica che esistono già delle decisioni del Tribunale federale concernente il personale che lavora nello studio medico. Il Comitato centrale pensa che sarà possibile trovare prossimamente una soluzione comune con santésuisse. Jürg Schlup si dichiara soddisfatto di questa informazione.

La proposta N. 7/2 (Charles Selleger, Ginevra) richiede al Comitato centrale, che nelle negoziazioni con gli assicuratori che operano nel sistema del terzo pagante (LAMal e LAINF), i pagamenti ai medici siano effettuati tramite il sistema PVR, quando il medico l'utilizza per le sue fatture (polizza di versamento arancione con numero di riferimento).

Poiché l'informatizzazione degli studi medici è stata resa obbligatoria, è illogico che gli assicuratori non adottino il sistema PVR, che semplifica la gestione elettronica dei dati nello studio medico. La proposta è approvata senza votazione dal Comitato centrale come mandato di negoziazione.

La proposta N. 7/3 (Beat Manser, Lucerna) auspica che il G7 sia incaricato di negoziare una ripartizione simmetrica del volume di compensazione. È scioccante che i cantoni con un livello dei costi molto basso all'inizio della fase di neutralità dei costi, poi di un livello medio che rimane inferiore alla media svizzera, siano «puniti» con dei valori di punto tariffari poco elevati. La proposta viene ritirata per motivi formali perché la Camera medica non può dare degli incarichi di negoziazione alle società cantonali. La proposta, tuttavia, viene trasmessa alla Conferenza dei presidenti cantonali che ha luogo lo stesso giorno affinché sia discussa.

\section{Codice di deontologia}

[trattato dopo il punto dell'ordine del giorno n. 11]

\subsection{Approvazione delle direttive dell'ASSM}

Sino ad ora esisteva un'unica direttiva relativa alle cure delle persone in fin di vita. Poiché questa direttiva non teneva sufficientemente conto 
della situazione pratica, Ursula Steiner-König, vicepresidente della FMH, spiega che si è proceduti a delle differenziazioni che hanno portato a quattro direttive in funzione di contesti diversi. La formazione dei medici interessati non viene certamente indicata in modo esplicito, ma è contenuta implicitamente nelle direttive.

\section{La proposta N. 8.1/1 (Comitato centrale)}

chiede che le quattro nuove direttive medicoetiche

- questioni dei limiti della medicina intensiva;

- trattamento e assistenza di pazienti di lunga durata con lesioni cerebrali importanti;

- trattamento e assistenza di pazienti anziani che necessitano di cure;

- trattamento e assistenza di pazienti in fin di vita

dell'Accademia svizzera delle scienze mediche (ASSM) per l'assistenza ai pazienti in fin di vita siano inserite nel codice di deontologia della FMH è approvata a grande maggioranza, con un voto contrario e 4 astensioni.

\section{Progetto HOP-Line}

[trattato dopo il punto dell'ordine del giorno $\mathrm{n}$. 7]

Degli ospiti sono presenti per questo punto dell'ordine del giorno: si tratta della dott.ssa Christiane Margairaz, del consiglio interdisciplinare per la medicina e la prevenzione della violenza (CIMPV), Ospedale universitario di Ginevra, e della dott.ssa Judit Pòk, medico capo al Policlinico ginecologico dell'Ospedale universitario di Zurigo.

Ursula Steiner-König, vicepresidente della FMH, introduce il punto all'ordine del giorno e inizia ricapitolando la decisione della Camera medica dell'11 ottobre 2003, che ha incaricato il Comitato centrale di trattare il problema della Professional Sexual Misconduct (PSM) e di sottoporre un progetto concreto alla Camera medica ordinaria 2005. A questo scopo, il Comitato centrale ha costituito un gruppo di lavoro in vista della realizzazione del mandato sotto la direzione di Werner Tschan. Questo gruppo ha presentato il suo rapporto nell'autunno 2004 e l'ha concluso per trasmetterlo alla Camera medica, completandolo con 17 raccomandazioni. In questi ultimi tempi, prima della riunione della Camera medica, i mass media hanno riportato diversi incidenti, a volte con molto fracasso, in relazione con la PSM e hanno indicato che que- sta Camera medica ne avrebbe discusso. A nome del Comitato centrale Ursula Steiner-König precisa in proposito che la nuova LPMed prevede che l'autorità cantonale di sorveglianza possa ordinare delle misure disciplinari. Già oggi, le commissioni di deontologia hanno dato al corpo medico delle possibilità di sanzioni che vanno sino alla pubblicazione nei giornali specializzati delle società mediche, compresa la FMH, comunicazione all'autorità interessata e agli assicuratori inclusa. Siccome la Svizzera al contrario degli Stati Uniti, per esempio - non conosce nessun obbligo di notifica dei medici colpevoli di PSM, il numero indicato dai mass media di 14000 casi circa all'anno non è credibile e ancora meno provato. Tutto questo fracasso è quanto mai deplorevole e proietta una luce sfavorevole su un argomento delicato e doloroso.

Nella sua presentazione, Christiane Margairaz spiega le conseguenze degli abusi da parte di medici nei confronti di pazienti: la persona ammalata diventa ancora più ammalata, in contraddizione, quindi, da un lato con il postulato di guarigione previsto dagli statuti, uno dei fondamenti del codice di deontologia e, dall'altro, distrugge la relazione di fiducia, e quindi la condizione di base per eccellenza dell'atto medico. Inoltre, l'insieme del corpo medico acquisisce una cattiva reputazione. L'obiettivo è quindi di attirare l'attenzione dei giovani medici sul fatto che non devono mai utilizzare la loro «posizione di forza» per soddisfare i loro bisogni e che non devono cercare la sessualità e l'erotismo nella relazione medico-paziente.

Judit Pòk riassume il contenuto del rapporto del gruppo di lavoro. Pur focalizzandosi sulla PSM, questo rapporto è tuttavia valido per tutte le forme di «cattiva condotta» professionale. Inoltre è chiaramente orientato verso un'offerta di sostegno per i medici - da cui il nome «HOP» abbreviazione di Help Our Physicians - sapendo che con un approccio del genere si viene implicitamente anche in aiuto dei pazienti. Il rapporto e le raccomandazioni in esso contenute vertono essenzialmente sulla consulenza individuale L'offerta proposta nell'ultima raccomandazione della lista consiste in una linea telefonica, un sito internet e un gruppo di specialisti a disposizione per la consulenza e l'assistenza. Il gruppo di specialisti dovrebbe essere ancora creato e le persone formate a questo scopo. Judit Pòk indica, tuttavia, che il gruppo di lavoro non esige imperativamente che si mantengano le raccomandazioni, ma che considera essenziale che la FMH tratti seriamente questo problema, che si distanzi chiaramente dalla cattiva condotta professionale 
al suo interno come anche all'esterno e che prenda delle misure concrete per la prevenzione e l'aiuto necessario.

Il rapporto del gruppo di lavoro e le raccomandazioni in esso contenute vengono apprezzate in modo diverso nel corso della discussione che segue; in particolare i dati quantitativi e qualitativi non possono essere seguiti. Gli oratori sono unanimi nell'affermare che alla PSM non deve essere accordato assolutamente nessun genere di tolleranza e che l'argomento deve essere trattato con la massima serietà. La proposta del gruppo di lavoro per una linea telefonica è problematica, perché non si sa se chi gestisce la linea incorra in sanzioni penali annunciando alle autorità i casi di cui si è venuti a conoscenza. Gli abusi sessuali sono dei delitti reprensibili. Inoltre, i delegati si chiedono se sia giudizioso investire per diversi anni delle somme importanti in un progetto prima di aver effettuato delle investigazioni per sapere se i medici implicati in una PSM telefoneranno o invieranno delle e-mail e quale sarà il numero all'anno. Sino ad oggi, al servizio di consulenza di Basilea non si è ancora annunciato nessuno.

\section{La proposta N. 9/1 (Comitato centrale)}

viene presentata da René Raggenbass, corresponsabile del settore «Salute e prevenzione». René Raggenbass costata che il Comitato centrale condanna totalmente gli abusi sessuali nella relazione medico-paziente, considerando che le proposte del gruppo di lavoro dell'HOP-Line non sono delle misure adeguate per risolvere il problema (linea telefonica alla segreteria generale? Sito Internet?) e che il rapporto con la realtà svizzera non è stato esaminato. Per questo motivo il Comitato centrale propone alla Camera medica di rinviare il progetto al gruppo di lavoro con l'incarico di elaborare un progetto pilota limitato a tre anni, realizzabile con un budget annuale di Fr. 10000.-. Il progetto pilota dovrà contenere delle proposte concrete di misure atte a combattere la PSM; queste misure dovranno essere vicine alla realtà ed essere sottoposte a test.

\section{Voto d'entrata in materia}

Dapprima la Camera si pronuncia sulla questione di sapere se si deve perseguire lo studio del dossier dedicato alla cattiva condotta professionale. I delegati si pronunciano chiaramente a favore con 3 voti contrari e 8 astensioni.

Il voto seguente oppone il progetto HOP-Line alla controproposta del Comitato centrale che viene approvata senza voti contrari e con 7 astensioni. La proposta del Comitato centrale viene quindi chiaramente approvata.

\section{Numero di chiamata d'emergenza valido in tutta la Svizzera}

[trattato dopo il punto all'ordine del giorno n. 8] Marc Müller, presidente della forum di salvataggio della FMH, informa sulle deliberazioni e sulle raccomandazioni del forum per quel che concerne il numero di chiamata d'emergenza (a tre cifre) valido in tutta la Svizzera: già nel 1996, nelle sue 12 tesi sul salvataggio pubblicate nel Bollettino dei medici svizzeri (in tedesco [1], in francese [2]), la FMH aveva richiesto nella sua tesi n. 2 l'installazione del numero di chiamata d'urgenza sanitaria $144 \mathrm{su}$ tutto il territorio svizzero, esente da tassa e come punto di contatto per tutte le situazioni d'urgenza medica. Il forum considera che questo postulato è ancora oggi appropriato e per questo motivo raccomanda di rinunciare al suo numero proprio a tre cifre per il servizio d'urgenza medica, perché creerebbe confusione. Il forum sta per elaborare delle basi decisionali contenenti una panoramica delle diverse forme d'organizzazione del servizio medico d'urgenza in Svizzera, all'attenzione delle società cantonali, dei governi cantonali competenti per organizzare il servizio d'urgenza e della CDS. L'IAS ha garantito la sua partecipazione.

\section{Concezioni di qualità del corpo medico svizzero}

[trattato dopo il punto all'ordine del giorno n. 9] In una breve introduzione Christoph Ramstein, il copresidente della VEDAG, spiega rapidamente i retroscena della proposta in discussione. HansAnton Vogel, membro del VEDAG, presenta le «linee direttrici del corpo medico svizzero in materia di qualità», sottoposte il 21 aprile 2005 ai presidenti delle organizzazioni rappresentate alla Camera medica svizzera e pubblicate nel Bollettino dei medici svizzeri (in tedesco [3], in francese [4]). Per l'iniziante è importante sottolineare che non si tratta di una questione interna del VEDAG, che il gruppo di lavoro proposto è aperto, che deve essere composto da rappresentanti di tutta la Svizzera e che si terrà conto delle conoscenze in materia, integrandovi degli esperti della Segreteria generale.

\section{Proposta N. 11/1 (Christoph Ramstein, VEDAG)}

La VEDAG propone la formazione di un «gruppo di lavoro qualità FMH», con l'incarico di a. sviluppare continuamente le linee direttive; 
b. incoraggiare la realizzazione del loro contenuto;

c. elaborare un concetto per un forum della qualità, per la formazione delle persone incaricate del settore della qualità e per la realizzazione di controlli della qualità;

d. iniziare delle negoziazioni con gli interlocutori sulla base delle concezioni del corpo medico svizzero in materia di qualità.

La proposta viene approvata con 5 voti contrari e 9 astensioni.

\section{HIN: collaborazione, progetti}

Rimandata alla Camera medica del 2.12.2005.

\section{Varie}

Nessuna.

Il presidente della FMH chiude la seduta alle 16.35 .

\section{Riferimenti}

1 Thesen der FMH zum Rettungswesen in der Schweiz. Schweiz Ärztezeitung 1997;78(12):421-2.

2 Thèses de la FMH sur le domaine du sauvetage en Suisse. Bull Méd Suisses 1997;78(14):496-7.

3 Peltenburg M, Kernen H, Schneider P, von Below GC, Waldis G, Vogel HA, et al. Qualität - ein Zusammenspiel aller Kräfte im ärztlichen Umfeld. Schweiz Ärztezeitung 2005;86(18):1055-8.

4 Peltenburg M, Kernen H, Schneider P, von Below GC, Waldis G, Vogel HA, et al. La qualité: une interaction de toutes les forces en présence dans le domaine médical. Bull Méd Suisses 2005;86(20):1216-20. 